\title{
Incorporation of computational creativity in arts education: Creative coding as an art course
}

\author{
Selcuk Artut ${ }^{1 \mathrm{a}}$ \\ ${ }^{1}$ Visual Arts and Visual Communication Design, Sabancı University, Istanbul, Turkey
}

\begin{abstract}
In the lights of the Information Renaissance, like the unavoidable transformation of everyday practices, contemporary educational perspectives and principles are going through a state of change. Recently, the proliferation of computer aided art education is widening its doors, because the contemporary artists are using computers not only as an assistive apparatus, but as a collaborative platform to infiltrate far beyond their imaginations and expressions. Therefore, the notion of creativity cannot be limited only to a human-centred approach, rather there is a significant need for discussing the discourse of dialectic creativity that embodies human technology collaborations. In this article, Computational Creativity is explored as an educational content for contemporary arts education with its implications and its various dimensions in the creative thinking process. In this study, a new course content called Creative Coding which is included in the curriculum of Sabanci University, Istanbul is explored as a proposed model to shed lights on the Computational Creativity. The course content and its methodologies will be explained while examining the technological dimensions in the art education.
\end{abstract}

Keywords: creativity, creative coding, arts education

\section{Introduction}

Art stands as one of the oldest phenomena on earth with its ambiguous nature full of uncertainties. Its underpinnings and origins are concealed in the history of civilization. Therefore, emphasizing the history of art and its evolution in time becomes an essential component in comprehensive arts education. However, by its nature history evolves and it never suspends. When history takes over the contemporary, the establishment debate initiates itself. During addressing an art student to enhance his/her knowledge and skills with educational materials, it is certain that the content also needs to be adjusted to the changes occurring in the contemporary society. When art is observed in different periods of time, it is inevitable that its discourse has been considerably influenced by the social movements and technological developments. Especially after the modern period in art, art is walking close hand in hand with the latest technological advancements in various

${ }^{\text {a }}$ Corresponding author: sartut@sabanciuniv.edu 
dimensions including kinetic art, video art, computer art etc. Several contemporary artists, whose numbers are notably increasing day by day, are creating unique art works with using new possibilities provided by technology.

Because of the Information Renaissance, with the use of Internet, we are exposed to a proliferation of information from various dimensions. Currently education systems are undertaking a structural transformation while considering the changing role of the educator as the primary source of influence. In this technological climate, to what extent should the art students be educated in this kind of technological exposition? How should the computational creativity be integrated in art education as a new apparatus to make distinct forms of art?

Today artists are not only using technology as an apparatus to enhance their artistic expressions, but to explore hidden dimensions in the formalization of art [1]. According to Flusser [2] we are going through a post-historical age with the extensive inclusion of Technical Images. As opposed to traditional paintings, by technical images Flusser includes works of photograph, films, videos, television screens and computer terminals of which all belong to a certain technological progress and innovation in recent history. These new forms of technological art disciplines have been widely acknowledged and utilized by the art scholars; therefore, the art education based on these disciplines have also been welcomed in art schools to a notable extent. While examining the contemporary methods in art education, the core subject of the ontological definition of art should be reconsidered under the existing conditions. We also need to address questions towards the role of the artist in the creativity process while using technological apparatuses.

\section{Exposition of computational art}

The computers become widely available to masses in the 1980s and onwards [3]. Back then there was a limited group of engineers and scientists who became experts in computer programming and their main purpose was to be dealing with solving complex problems in their field of expertise. In the following years when game industry was to take a leap towards becoming a powerful attraction for the computer users, computers became a medium of interest for expressing art into digital forms. Many game developers started to not only focusing on the game logic but also interested in building visually appealing elements. However, the game industry can be considered only as one component of the related influences on computational art. Additionally we may include some procedural art making examples in the forms of performance art, music, painting etc. that are taking place in the 1950s and onwards. As an example, in Alvin Lucier's famous performance (1969) "I am sitting in a room" we are observing Lucier to repeatedly recording himself inside a studio while replaying the previous recording of himself. This performance illustrates a recursive structure which is widely observed in most computational art pieces. In the same manner, recursive algorithms are widely observed in Computer Programming implementations such as fractals, l-tree systems, etc. [4]. Likewise, some computer programmers were interested in creating algorithms that would simulate and replicate a form of nature in a programming environment, and some contemporary artists were also significantly influenced by these automatic creative skills built by computational operations. Even though it is not possible to track the earliest, Frieder Nake, Manfred Mohr, George Nees, John Whitney are regarded as early pioneers in digital computer art who were exhibiting their art pieces in acknowledges venues. According to Francesca Franco, computer art was introduced in the mid-1950s to early 1960 s as a mainstream genre $[5,6]$. Individual examples of using digital computers and programming to create works of art may follow, but Cybernetic Serendipity exhibition that took place at the Institute of Contemporary Arts (ICA) in London (1968) would be considered as one of the early 
examples for a group show that gathers several artists who are affiliated with the use of computation in their creation of artworks.

With the influence of Information Renaissance, computational forms have been inserted in the traditional art forms. Therefore, some main art attractions such as biennials, art fairs and museums such as Art Basel, Art Hong Kong have started to welcome art works with computation influences. Alternatively, we may also observe the new formation of art foundations and events focusing around the theme of computational creativity with announcing names such as Computer Art, New Media, Electronic Arts, Media Arts etc. Here below is a brief list of most well-known events organized around the idea of computational art forms:

Table 1. Acknowledged computational art events, festivals and exhibitions.

\begin{tabular}{|c|c|}
\hline Name of the Event & Location \\
\hline ISEA International Symposium on Electronic Art & in various countries \\
\hline Prix Art Electronica & Austria \\
\hline Kikk Festival & Belgium \\
\hline Enter Festival & Czech Republic \\
\hline Transmediale & Germany \\
\hline Dutch Electronic Art Festival & The Netherlands \\
\hline Resonate & Serbia \\
\hline Sonar & Spain \\
\hline One dot Zero & UK \\
\hline File & Brazil \\
\hline Sight and Sound & Canada \\
\hline New York Electronic Arts Festival & USA \\
\hline Japan Media Arts Festival & Japan \\
\hline
\end{tabular}

When art is to be integrated with technological factors, physicality of art needs to be reconsidered within the limits of its reflections. For instance, if the art considered reflects 
its properties in the digital realm with using software only, it becomes immaterial to a certain degree. The artworks' exhibiting standards are to be reconsidered as well. According to Xiaobo [7] when we are talking about an interaction between a human and a system three aesthetic criteria needs to be elaborated; 1) Addressing to all senses, not only visually 2) Extension of Temporal Relations 3) Comprehensive Experience. As a result, if we are to be introducing use of technology or to be more specific use of computation, the traditional frameworks in art education needs to be reconsidered extensively.

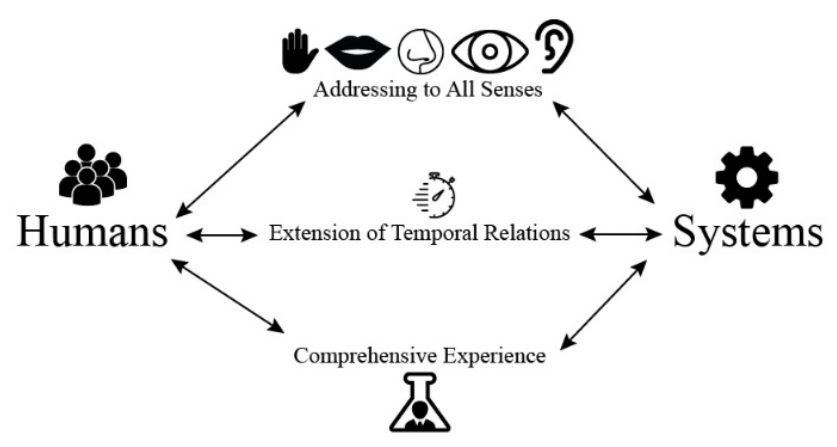

Fig. 1. Xiabo's interaction framework between a human and a system

\section{Teaching computational skills for art students}

In the recent years, there has been a wide interest in building interdisciplinary curriculums in the education programs. According to World Economic Forum's Report [8] on 10 skills you need to thrive in the Fourth Industrial Revolution, "Creativity" has been listed as the 10th in year 2015, successively "Creativity" has been listed as the 3rd for the year 2020 . One aspect of such a need is that the new generations are heavily bombarded with technological tools, and computer becomes a common ground to integrate multiple skills in a single environment. Therefore, we have turned out to be rapid task switchers or multitaskers while working on our engagements. Students have eager to explore various interests and a standardized education system has difficulties in accommodating these demands. Same issues would be addressed to art education as well. Using computational skills in the process of art making is a contemporary issue. However, there is an increasing interest in teaching computational creativity skills in various schools of visual art, music, design and architecture [9]. Furthermore, creativity is not only treated as a means of human only production.

When Sabanci University in Istanbul was established in 1994 its philosophy was based on adopting an interdisciplinary approach of "learning to learn" to cultivate knowledge, support education and facilitate social advancement [10]. In the same manner, Visual Arts and Visual Communication Programme offers interdisciplinary courses to students from various disciplines including humanities and arts, social sciences, and natural sciences. Therefore, class environment becomes a medium where people from different interests are met and new explorations are to be foreseen. In this article, a new course opening titled VA345 Creative Coding is to be introduced and its content will be elaborated below. 


\section{Building a creative coding course}

VA345 Creative Coding course explores code at a conceptual level with the aim of using computation as an expressive and creative tool. It addresses topics such as generative audio-visuals, computational art, scripting for common media tools. The structure is based on experiential learning with utilizing projects on themes. Students who have backgrounds and needs from various disciplines are treated equivalent in terms of their abilities and skills. Here below is the weekly course content;

Table 2. VA345 Creative Coding Weekly Contents.

\begin{tabular}{|c|c|}
\hline Teaching Period & Course Content \\
\hline Week 1 & Introduction, Course Objectives, Students' Expectations \\
\hline Week 2 & Automatons \\
\hline Week 3 & Algorithmic Forms in Art \\
\hline Week 4 & Processing.org Programming Platform \\
\hline Week 5 & Conditions, Iteration, Looping \\
\hline Week 6 & Arrays, Lists, Classes \\
\hline Week 7 & Midterm Project \\
\hline Week 8 & Typographical Design by Coding \\
\hline Week 9 & Fractals / L-Tree Systems / Recursions \\
\hline Week 10 & Particle Systems \\
\hline Week 11 & Playing with Sound \\
\hline Week 12 & Computer Vision \\
\hline Week 13 & Exhibition Production \\
\hline Week 14 & Exhibition Production \\
\hline
\end{tabular}


VA 345 Creative Coding assumes coding not only as a programming paradigm on a flat screen, but rather it aspires to explore computational thinking in all reflections including even building physical systems with no use of computer at all. Each week a new Computational Artist's artworks are brought to class to familiarize students with contemporary examples. Works of artists such as Joshua Davis, Aaron Koblin, Casey Reas, Sougwen Choung, Joachim Sauter, Theo Jansen, Karsten Schmidt, Golan Levin and Alexandor Randon will be discussed during the class hours. As an introduction finding a proper definition for Creativity is examined, thus following questions would arise including "How do we incorporate creativity in computational disciplines?". To establish strong links to find out these inquiries, a history of Computational Arts is studied with watching Casey Reas' Gray Area Festival presentation "History of the Future, Art \& Technology from 1965 - Yesterday". Second week focuses on Automatons as self-operating systems. Since automatons are built to be following calculated set of rules to succeed an operation, their systems are of interest for analysing the determination of a procedure to be utilized. History of Automatons is introduced by screening "Pierre Jacquet-Droz's Androids" documentary. Mark Sussman describes automatons as dramaturgical hybrid of theatre, magic, and science, similarly the subject is an essential component of how a mental labour is transformed into a mechanization [11]. To exemplify the creative process behind the automatons, the students are given a automaton project to be built with use of simple cardboards and handcraft tools. Next theme in the course content is Algorithmic Forms in Art. During this week students are introduced to several examples of how algorithm turns ideas into complex, abstract visuals. Even though examples presented will be outcomes of programming frameworks, the students will still not be introduced to computer programming environments until the fourth week. On the contrary, the students will be required to build a mechanical Harmonograph with use of wooden structures. With the use of oscillations in its movements, Harmonograph creates complex geometric images. In week 4, students are introduced to gain basic programming skills with the use of Processing Programming Framework. Processing embodies software paradigms to handle creation of visual form, motion and interaction [12].

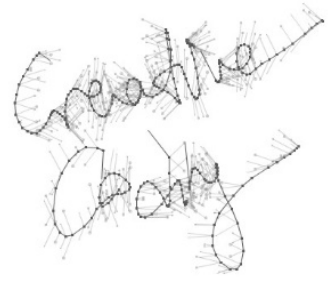

Fig. 2. First steps in building a computational system

When basic insight is introduced, students will be motivated to construct more complex projects with the use of Loops, Iterations, Arrays, Lists and Conditionals. Next in the agenda typographic elements will be explored and an assignment based on building a design project will be provided. Students will be informed to learn the main principles and properties that construct a type-face so that they would be able to deconstruct and construct experimental approaches to accomplish artistic tasks. Later in the course Fractals, L-Tree Systems will be studied as a recursive approach towards creating complex forms with the use of simpler ideas. In week 10, the particle systems are elaborated with discussions evolving around multiplication and pattern registration. Basic sound use is included to 
facilitate students to experiment with use of sound via computational frameworks. Finally, how computers perceive the world with use of image processing is investigated with computer vision theories. As a result, participants will be able to use basic image processing skills such as motion detection, skeleton tracking, blob detection to hunt for ideas to create artistic projects. At the end of the course, a collective art exhibition project is organized to have the participants experience the process of exhibit preparation and display their artworks.

\section{Conclusion}

Computational Art has driven the art educators to question possible uses of computers in the arts. Even though artistic pieces have a certain kind of aesthetic framework, recently extensive use of technology has been enrolled as a major factor in determining the new aesthetical formations in solidifying artistic ideas into shapes and forms. In the light of all these discussions, an adaptive education system based on experiencing computational skills in art making needs to be considered in various dimensions. In this article, VA345 Creative Coding course has been exposed as an effort to examine the barriers and opportunities between art and computational sciences. Students participating this course will not only be acknowledging the field as a contemporary development to be aware of, but to gain experience in the making of the art projects.

\section{References}

1. Truckenbrod, J., A New Language for Artistic Expression: The Electronic Arts Landscape. Leonardo 21(5) 99-102, (1988)

2. Flusser, V., Into the universe of technical images. 32. U of Minnesota Press, 2011.

3. King, R. G., Computer graphics and animation as agents of personal evolution in the arts. Leonardo. Supplemental Issue 43-46, (1988)

4. Saupe, D., Algorithms for random fractals. The science of fractal images. Springer New York, 71-136, (1988).

5. Franco, F., Computational art exhibitions in London, 2014: A New Hope." Visual Resources 31(3-4) 201-210, (2015)

6. Prince, P. D., The Aesthetics of Exhibition: A Discussion of Recent American Computer Art Shows. Leonardo 21(5) 81-88, (1988)

7. Xiaobo, L., and Liu Y., Embodiment, Interaction and Experience: Aesthetic Trends in Interactive Media Arts. Leonardo 47(2) 166-169, (2014)

8. https://www.weforum.org/agenda/2016/01/the-10-skills-you-need-to-thrive-in-thefourth-industrial-revolution/ (Retrieved 14.05.2017)

9. Bergstrom, I., and R. Beau Lotto, Code Bending: A new creative coding practice. Leonardo (2015).

10. http://www.sabanciuniv.edu/en/about-sabanci/philosophy (Retrieved 14.05.2017)

11. Nake, F., and Susanne G., Human-computer interaction viewed as pseudocommunication. Knowledge-Based Systems 14(8) 441-447, (2001)

12. Reas, C., and Fry, B., Processing: a programming handbook for visual designers and artists. No. 6812. Mit Press, (2007). 